\title{
ROLE OF MACROLIDES (CLARITHROMYCIN) ON BIOFILMS IN CHRONIC RHINOSINUSITIS
}

Authors: Mohd Aftab (1), Sachin Jain (2), Daya Shanker (3), Mohd Rizvan (4), Pankaj KumarTiwari (5)

Authors Affiliations: (1) Associate Professor, (2) Professor and Head, (3) Junior Resident, 94) Junior Resident, (5) Junior Resident, Department of ENT \& Head Neck Surgery, M.L.N. Medical College, Prayagraj, Uttar Pradesh, India

\begin{abstract}
Background: Rhinosinusitis, a group of disorders characterized by inflammation of the nose and paranasal sinuses can be classified as acute, sub-acute or chronic and recurrent based on the duration of inflammation. Biofilm with formation of a physical barrier prevent antibiotics from penetrating it. Macrolides achieve high intracellular concentration and have a spectrum of activity against Gram positive cocci. This study aims to describe effect of macrolide antibiotics (clarithromycin) on eradication of suspected biofilms in chronic rhinosinusitis.
\end{abstract}

Methods: Fifty ( 29 man and 21 women) patients of Chronic Rhinosinusitis were prospectively observed and data were collected.

Results: The average age of Chronic Rhinosinusitis onset was 28.6 years. Suspected biofilms was noted in all the patients. The case group showed a reduction of $25.8 \%$ in symptom score and $49.2 \%$ in endoscopic sign scores with clarithromycin.

Conclusions: The case group showed a reduction of bacteriologically as well as symptom score and endoscopic sign scores.

Keywords: Chronic Rhinosinusitis, Biofilm, Clarithromycin

\section{Introduction}

Rhinosinusitis is defined as a group of disorders characterized by inflammation of the nose and paranasal sinuses. Based on the duration of inflammation, rhinosinusitis is classified as acute
(< 4 weeks), subacute (4-12 weeks) or chronic ( $>12$ weeks), recurrent [ $>4$ episodes of Acute Rhino Sinusitis peryear].

CRS is classified into CRS with nasal polypand CRS without nasal polyp.Symptoms of CRS have been classified as major (facial pain or pressure, nasal obstruction or blockage, discoloured postnasal drainage, hyposmia and purulence in nasal cavity) or minor(headache, fever, dental pain and ear pain). ${ }^{1}$

Biofilms are a structurally complex, three dimensional community of bacteria anchored to a surface. Individual bacterial within a biofilm are connected by a self-extracted matrix consisting of negatively charged polysaccharide. Once organized, biofilms are capable of evading both the effects of antibiotics as well as host immunologic defense systems. ${ }^{2}$ Some CRS patients appear resistant to cure despite surgically opened sinus cavities and long-term culture-directed antibiotic therapy.

Several studies ${ }^{3,4,5,6}$ have investigated the relationship between biofilms and CRS. The most common bacterial biofilms in CRS are Staphylococcus aureus, Pseudomonas aeruginosa, coagulase negative Staphylococci, Streptococcus pneumoniae, Moraxella catarrhalis and Haemophilusinfluenzae.

Macrolides belong to the family of 14 or 15 membered lactone ring antibiotics. These antibiotics achieve high intracellular concentration and have a spectrum of activity against Gram positive cocci ${ }^{7}$

Macrolides can block the activation of 
transcription factor nuclear factor $\mathrm{kB}$ and inhibit the production of various inflammatory cytokines, including interleukin (IL)-8 and tumor necrosis factor- $a$ (TNF- $\alpha$ ). They can also suppress the secretion of airway mucus, induce the apoptosis of neutrophils, and even diminish the formation of bacterial biofilms. ${ }^{8,9}$

This study was planned to obtain the evidence of suspected biofilms in chronic rhinosinusitis and effect of macrolide antibiotics (clarithromycin) on eradication of suspected biofilms in chronic rhinosinusitis.

\section{Methods}

This prospective, Observational study was conducted in the Department of E.N.T., MotiLal Nehru Medical College and Swaroop Rani Nehru Hospital, Prayagraj from September 2018 to August 2019.

\section{Inclusion Criteria}

Age group of 18 - 45 years irrespective of Gender, patients with diagnosed chronicsinusitis [radiological examination and DNE(diagnostic nasal endoscopy)], two or more of the following symptoms (Anterior/ posterior mucopurulent nasal discharge, Nasal obstruction, Facial pain/ pressure/ fullness) persisting since $>12$ weeks, endoscopy signs: discolored mucus or edema within the Middle meatus or Ethmoid Region, evidence of Rhinosinusitis on CT Scan, patients who were willing to come for follow up and patients of CRS not responding to treatment even after three months with commonly used macrolides prescribed at $\mathrm{CHC}, \mathrm{PHC} \&$ by general physician were considered as a case of having suspected biofilm.

\section{Exclusion Criteria}

Patients having no other sinonasal pathology like polyposis malignancy, fungal rhinosinusitis, frontal mucocoele and deviated nasal septum, patients of comorbid conditions like diabetes mellitus, hypertension, psychological issues, liver disease, immunocompromised, patients with pregnancy or lactation, history of long term use of systemic steroid therapy and patient of CRS with nasal polyp

Clinical data including gender, age, symptoms, systemic disorders and related medications were collected. Patients were properly examined and selected for our study who fulfilled the criteria by International Advisory Board, $1997{ }^{10}$ andthe diagnosis of chronic rhinosinusitis was made.

Patients of age group 18 - 44 years with symptoms and signs of Chronic Rhino-sinusitis were randomized into two treatment groups.Group $A(n=25)$ were treated with macrolides antibiotics (Clarithromycin) and Group B $(n=25)$ were treated with a placebo.

Swab was taken in both the groups from middle meatus for study of bacteriological spectrum of suspected biofilm. The isolated pathogens were-examined microscopically to ensure the staining and morphologic characteristic of an organism. Further identification was done at species level by the Vitek-2 system.

After diagnosis of CRS with suspected bacterial biofilms. In Group A Oral Clarithromycin 500 mg BD was administered for 2 weeks followed by $250 \mathrm{mg}$ OD for next 6 weeks and in Group B placebo was administered.

Quality of life assessment pre-treatment as well as post-treatment was done by using Visual Analogue Score (VAS) at regular intervals of 0 , 2 weeks and 6 weeks. ${ }^{11}$

Nasal endoscopic scoring was also done before starting of treatment and at regular intervals after starting of treatment (i.e. at 0, 2 weeks, 6 weeks) according to Modified Lund-Kennedy Endoscopy Scoring system ${ }^{12}$.

\section{Results}

All fifty ( 29 man and 21 women) patients with 
mean age 28.6 years of suspected biofilms were found positive for aerobic as well as anaerobic bacteria.

Table-1: Comparison of microorganism profile of two study groups

\begin{tabular}{|l|c|c|c|}
\hline Pathogens isolated & Group-A & Group-B & Total \\
\hline Peptococcus & $9(36.0 \%)$ & $5(20.0 \%)$ & $14(28 \%)$ \\
\hline S. aureus & $16(64.0 \%)$ & $20(80.0 \%)$ & $36(72 \%)$ \\
\hline Total & $25(100 \%)$ & $25(100 \%)$ & \\
\hline
\end{tabular}

In Group A, a total of 16(64\%) were S. Aureusand $9(36 \%)$ were Peptococcus whereas in Group B, 20 ( $80 \%)$ were S. aureus and $5(20 \%)$ were Peptococcus. Though the proportion of Peptococcuswas slightly higher in Group A as compared to that in Group B.

Table No-2: Comparison of clarithromycin sensitivity in both study group

\begin{tabular}{|l|c|c|c|}
\hline Clarithromycin & Group-A & Group-B & Total \\
\hline Sensitivity & $20(80.0 \%)$ & $22(88.0 \%)$ & $42(84 \%))$ \\
\hline Resistant & $5(20.0 \%)$ & $3(12.0 \%)$ & $8(16 \%)$ \\
\hline Total & $25(100 \%)$ & $25(100 \%)$ & \\
\hline
\end{tabular}

$c^{2}=0.595 ; p<0.440$

On evaluating the sensitivity of Clarithromycin, it was found to be sensitive in $42(84 \%)$ cases, while $8(16 \%)$ cases were resistant $(2 \%$ S.aureus and $14 \%$ Peptococcus).

In Group A, mean endoscopic sign scores for discharge were $2.56 \pm 0.77,1.64 \pm 0.49$ and $1.40 \pm 0.71$ respectively at baseline, first and second follow-up intervals. As compared to baseline, there was a decline of $0.92 \pm 0.91$ $(35.9 \%)$ at first and $1.16 \pm 1.03(45.3 \%)$ at second follow-up intervals. $(p<0.001)$.

In Group B, Mean endoscopic sign scores for discharge were $2.44 \pm 0.77,2.52 \pm 0.59$ and $2.44 \pm 0.58$ respectively at baseline, first and second follow-up intervals. As compared to baseline, there was an increase of $0.08 \pm 0.81$ (3.3\%) at first follow-up while no change was observed at second follow-up.

At baseline in Group A, a total of 20 (80\%) cases was sensitive and while 5 (20\%) were resistant, in Group B, 22(88\%) cases were sensitive and $3(12 \%)$ were resistant against Clarithromycin.

Table 3: Evaluation of Change in Symptom scores as compared to baseline at different

\begin{tabular}{|c|c|c|c|c|c|c|}
\hline \multirow[t]{2}{*}{ Group } & \multirow[t]{2}{*}{$\begin{array}{c}\text { Follow-up } \\
\text { interval }\end{array}$} & \multirow[t]{2}{*}{ Mean \pm SD } & \multicolumn{2}{|c|}{ Improvement } & \multicolumn{2}{|c|}{$\begin{array}{c}\text { Significance of } \\
\text { change (Paired } \\
\text { 't'-test) }\end{array}$} \\
\hline & & & $\operatorname{Mean} \pm$ SD & $\begin{array}{c}\% \\
\text { Change }\end{array}$ & ' $t$ ' & 'p' \\
\hline \multirow[t]{3}{*}{ A } & Baseline & $40.64 \pm 3.03$ & - & - & - & - \\
\hline & First & $37.68 \pm 2.50$ & $2.96 \pm 3.06$ & $7.3 \%$ & 4.834 & $<0.001$ \\
\hline & Second & $30.16 \pm 1.80$ & $10.48 \pm 3.50$ & $25.8 \%$ & 14.965 & $<0.001$ \\
\hline \multirow[t]{3}{*}{ B } & Baseline & $39.44 \pm 2.99$ & - & - & - & - \\
\hline & First & $38.64 \pm 2.23$ & $0.80 \pm 1.83$ & $2.0 \%$ & 2.191 & 0.038 \\
\hline & Second & $37.84 \pm 2.66$ & $1.60 \pm 0.91$ & $4.1 \%$ & 8.764 & $<0.001$ \\
\hline
\end{tabular}

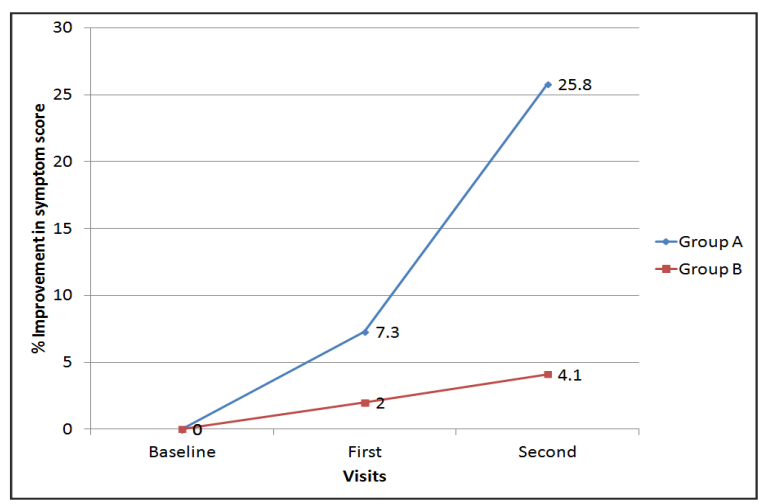

follow-up intervals in two study groups

Fig. 1 : \% Improvement in symptom scores at different time intervals in Groups A and B In Group A, mean symptom score was $40.64 \pm 3.03,37.68 \pm 2.50$ and $30.16 \pm 1.80$ respectively at baseline, first and second followup intervals respectively. As compared to baseline, there was a decline of $2.96 \pm 3.06(7.3 \%)$ at first follow-up and $10.48 \pm 3.50(25.8 \%)$ at second follow-up. Statistically, the change from baseline was significant at both the follow-up intervals $(p<0.001)$.

In Group B, mean symptom score was $39.44 \pm 2.99,38.64 \pm 2.23$ and $37.84 \pm 2.66$ respectively at baseline, first and second followup intervals respectively. As compared to 
baseline, there was a decline of $0.80 \pm 1.83(2 \%)$ at first follow-up and $1.60 \pm 0.91$ (4.1\%) at second follow-up. In statistical terms, the change from baseline was highly significant at both the follow-up intervals $(p<0.05)$.

\section{Discussion}

Chronic rhinosinusitis is a multidimensional disease having a huge economic burden, morbidity and quality of life deterioration. Evidence has shown that microorganisms play a crucial role in aggravating the symptoms and mucosal inflammation Boaseet al., 2013 ${ }^{13}$. The characterization of biodiversity of microorganisms in CRS could be done by carrying out microbiological assessment of potential specimen that could help in identifying and characterizing it.

In this context biofilms are an important source, for they have a high potential of diverse microbiological species at one place, moreover they represent the high possibility for easy isolation and identification of microbial organisms. Biofilms are highly organised microbial communities enclosed within a selfproduced extra cellular polymeric matrixHallStoodleyet al., 2004 ${ }^{14}$. There are limited studies on characterizing the biofilms in chronic rhinosinusitis patients.

Macrolides are broad spectrum antibiotics that include antibiotics like clarithromycin, azithromycin and erythromycin. They have been shown to have a successful role in inhibition of bacteria. Hence, the present study was carried out with an aim to identify the bacteriological spectrum in suspected biofilms of chronic rhinosinusitis and to assess the clinical efficacy of macrolide antibiotic (Clarithromycin) on these suspected biofilms. For this purpose, a total of 50 ( 29 man and 21 womens with mean age 28.6 years) chronic rhinosinusitis patients without polyps were enrolled in the study.

In present study, out of a maximum possible score of 60 , the VAS scores of patients ranged from 34 to 46 with a mean of $40.04 \pm 3.04$. These scores thus indicated a moderate burden of disease. Mean scores for discharge and edema were $2.50 \pm 0.76$ and $2.66 \pm 0.80$ respectively out of a maximum of 4 for each domain. Overall mean total sign score was $5.16 \pm 1.02$ which also reflected a moderate burden of disease for the two domains remaining in the study. Thus both sign and symptom scores were reflective of a moderate burden of disease. In present study, the bacteriological profile of suspected biofilms indicated a dominance of Staphylococcus aureus $(n=36 ; 72 \%)$.

In present study a significant improvement in signs was observed only in case group and it had a significant difference from control group from first visit itself. On final follow-up, the case group showed a reduction of $25.8 \%$ in symptom score and $49.2 \%$ in endoscopic sign scores. These results are similarly withLuoet $\mathbf{a l . 2 0 1 4}{ }^{15}$, who reported a reduction of $64.5 \%$ in symptom score and $49.5 \%$ in endoscopic sign scores. Deng et al.2018, ${ }^{16}$ in a randomized controlled trial found that symptomatic results are slightly better in long-term, low-dose with Clarithromycin group as compared to control group. Sarfaraz et al.,2008; ${ }^{17}$ Fan et al., 2014; ${ }^{18}$ Sireci et al., $2018^{19}$ and Wallwork et al., $2006^{20}$ also found similar results with Clarithromycin.

The findings of present study, thus show a promising role of clarithromycin in treatment of chronic rhinosinusitis without polyps both in terms of symptomatic response as well as antibacterial effect.

\section{Conclusions}

The present study was a novel to describe the bacteriological spectrum of biofilms of chronic 
rhinosinusitis patients and to assess the clinical efficacy of macrolide antibiotics (Clarithromycin) on these biofilms. This study also showed a significant curative effect of macrolide antibiotic (Clarithromycin) clinically and symptomatically as well as bacteriologically. The significance of this study is incidence of anerobes especially peptococcusfrom the suspected biofilm which were never reported before.

\section{References}

1. Benninger MS, Ferguson BJ, Hadley JA, Hamilos DL, Jacobs M, Kennedy DW, et al. Adult chronic Rhinosinusitis: Definitions, Diagnosis, Epidemiology, and Pathophysiology. Otolaryngology Head and Neck Surgery. 2003;129(3):S1S32.

2. Stewart PS. Costerton JW: Antibiotic resistance of bacteria in biofilms. Lancet 2001;358:135-138.

3. Hunsaker DH, Leid JG. The Relationship of B i ofilms to Chronic Rhinosinusitis.Current Opinion in Otolaryngology \& Head \& Neck Surgery. 2008;16(3):237-41.

4. Ramadan HH. Chronic Rhinosinusitis and Bacterial Biofilms.CurrentOpinion in Otolaryngology \& Head \& Neck Surgery. 2006;14(3):183-6.

5. Suh JD, Cohen NA, Palmer JN. Biofilms in Chronic Rhinosinusitis. Current Opinion in Otolaryngology \& Head \& Neck Surgery. 2010;18(1):27-30.

6. Cohen $\mathrm{M}$, Kofonow J, Nayak JV, Palmer JN, Chiu AG, Leid JG, et al. Biofilms in Chronic Rhinosinusitis: A Review. American Journal of Rhinology \& Allergy. 2009;23:255-60.

7. Cervin A, Wallwork B. Macrolide therapy of chronic rhinosinusitis. Rhinology.2007;45(4):259.

8. Zeng M, Li ZY, Ma J, Cao PP, Wang H, Cui $\mathrm{YH}$, Liu Z. Clarithromycin and dexamethasone show similar antiinflammatory effects on distinct phenotypic chronic rhinosinusitis: an explant model study. $B M C$ immunology.2015;16(1):37.

9. Tamaoki J. The effects of macrolides on inflammatorycells.Chest.2004;125(2):41 S-51S.

10. International Rhinosinusitis Advisory Board. Infectious rhinosinusitis in adults: classification, etiology and management. Ear Nose Throat J. 1997; 76:1-22.

11. VAS by Lund VJ and Mackay IS. Surgical management of sinusitis. Scott Brown's Otolaryngology 2008, 7th edition, ; Edward Arnold publishers, Page 1490; Table 117.9.

12. Psaltis AJ, Li G, Vaezeafshar R, Cho KS, Hwang PH. Modification of the LundKennedy endoscopic scoring system improves its reliability and correlation with patient-reported outcome measures. Laryngoscope. 2014; 124(10):2216-2223.

13. Boase S, Foreman A, Cleland E, Tan L, Melton-Kreft R, Pant $\mathrm{H}, \mathrm{Hu} F Z$, et al. The microbiome of chronic rhinosinusitis: culture, molecular diagnostics and biofilm detection. BMC Infectious Diseases 2013; 13:210.

14. Hall-Stoodley L, Costerton JW, Stoodley P. Bacterial biofilms: from the natural environment to infectious diseases. Nature Reviews Microbiol. 2004;2(2):95-108.

15. Luo Q, Deng J, Xu R, Zuo K, Li H, Shi J. 
Clinical effect of clarithromycin therapy in patients with chronic rhinosinusitis. Chinese Journal of Otorhinolaryngology Head And Neck Surgery. $2014 ; 49(2): 103-8$.

16. Deng J, Chen F, Lai Y, Xu R, Ou C, Fu Q, Shi J. Lack of additional effects of long-term, low-dose clarithromycin combined treatment compared with topical steroids alone for chronic rhinosinusitis in China: a randomized, controlled trial. International Forum of Allergy\& Rhinology. $2018 ; 8(1): 8-14$.

17. Sarafraz $M$, Khosravi $A D$, Ahmadi K. Clinical and Microbiological Evaluation of Long Term Clarithromycin in the Treatment of Chronic Rhinosinusitis. Journal of Medical Sciences 2008; 8(7): 669-72.

18. Fan $\mathrm{Y}, \mathrm{Xu} \mathrm{R}$, Hong $\mathrm{H}$, et al. High and low doses of clarithromycin treatment are associated with different clinical efficacies and immunomodulatory properties in chronic rhinosinusitis. Journal of Laryngology\& Otology. 2014;128:236-241.

19. Sireci $F$, Speciale $R$, Gallina S, Sorrentino $\underline{R}$, Canevari FR. Clarithromycin in the Management of Chronic Rhinosinusitis: Preliminary Results of a Possible Its New Use. Indian Journal Otolaryngology Head Neck Surgery. $2018 ; 70(1): 87-91$.

20. Wallwork B, Coman W, Mackay-Sim A, Greiff L, Cervin A. A double-blind, randomized, placebo controlled trial of macrolide in the treatment of chronic rhinosinusitis.Laryngoscope.2006;116(2 ):189-93
${ }^{*}$ Corresponding author:

\section{Dr. Daya Shanker}

Junior Resident,

Department of ENT \& Head Neck Surgery,

M.L.N. Medical College, Prayagraj,

Uttar Pradesh, India.

Phone (Mobile) No. +918923070901

Email: orljournal.ald@gmail.com 